\title{
Classical novae in outburst: evolution of the ultraviolet emission lines in $\mathrm{CO}$ novae
}

\author{
A. Cassatella ${ }^{1,2}$, A. Altamore ${ }^{2}$, and R. González-Riestra ${ }^{3}$ \\ 1 INAF, Istituto di Fisica dello Spazio Interplanetario, Via del Fosso del Cavaliere 100, 00133 Rome, Italy \\ e-mail: cassatella@fis.uniroma3.it \\ 2 Dipartimento di Fisica E. Amaldi, Università Roma Tre, Via della Vasca Navale 84, 00146 Rome, Italy \\ 3 XMM Science Operation Centre, ESAC, Villafranca Satellite Tracking Station, PO Box 50727, 28080 Madrid, Spain
}

Received 12 January 2005 / Accepted 11 March 2005

\begin{abstract}
A comparative study is made of the temporal changes of the ionization level in seven CO novae well monitored with the IUE satellite at low resolution. The investigation is based on the evolution of the fluxes in eight strong ultraviolet emission lines covering a wide range of ionization potentials, from $\mathrm{Mg}$ II $(7.6 \mathrm{eV})$ to $\mathrm{NV}(77.5 \mathrm{eV})$. We find that the rate of increase of the ionization conditions in the emitting regions is uniform in the sample of novae considered, provided the time scale is normalized to the optical decay time $t_{3}$. The implications of this result for the nova ejected masses and for the nuclear turnoff time are discussed.
\end{abstract}

Key words. stars: novae, cataclysmic variables - techniques: image processing - ultraviolet: stars - methods: data analysis

\section{Introduction}

In a previous paper (Cassatella et al 2002, hereafter Paper I) we studied the evolution of the ultraviolet continuum and of the $\mathrm{O}$ I $1300 \AA$ emission line flux in 12 classical novae, selected among those best monitored at low resolution with the IUE satellite. The nova sample contained objects with optical decay time $t_{3}$ in a wide range from 5 to 100 days, and namely four Neon novae and eight CO novae, including V705 Cas, known for having undergone a spectacular dust formation process. In spite of the heterogeneity of the sample, we found that the time evolution of the UV continuum and of the O I $1300 \AA$ line flux is substantially homogeneous for all objects, provided the time scale is normalized to the $t_{3}$ decay time which, according to Livio (1992) is primarily an indicator of the white dwarf mass.

This homogeneous behaviour has been inferred from data obtained while the novae were bright enough in the UV continuum and in the $\mathrm{O} \mathrm{I}$ emission line, i.e. during a time of the order of $t_{3}$. To look for additional physical parameters, apart from the white dwarf mass, affecting the long term evolution of novae in the UV, one should analyse the evolution of the emission lines, which remain strong during a time that is typically much longer than the $t_{3}$ time.

In the present paper we study the evolution of the UV emission lines for seven CO novae from Paper I, leaving Neon novae apart for a separate investigation. Our main purpose is to verify to whath extent the time evolution of the emission lines is homogeneous for $\mathrm{CO}$ novae.

\section{Observations}

The relevant parameters of the seven $\mathrm{CO}$ novae selected for this study are given in Table 1 which contains, for each object, the day of the discovery $t_{\text {disc }}$, the time of visual maximum $t_{0}$, the visual magnitude at maximum light $V_{\max }$, and the optical decay time $t_{3}$, defined as the time to fade 3 mag below visual maximum.

A total of 411 low resolution large aperture spectra were obtained with IUE for these objects. These data were retrieved through the INES (IUE Newly Extracted Spectra) archival system (see e.g. http://ines. laeff.esa.es), which also provides the detailed log of the observations.

To study the time evolution of the ionization conditions in the nova envelopes we have chosen eight emission lines covering a wide range of ionization potential from $\mathrm{Mg}$ II $(7.6 \mathrm{eV})$ to $\mathrm{NV}(77.5 \mathrm{eV})$. In the case of the O I $1300 \AA$ line, the ionization potential has been taken to be $13.6 \mathrm{eV}$, since the $\mathrm{O}$ I line is pumped by fluorescence of Lyman $\beta$ and therefore reflects the strength of the Lyman $\beta$ emission (see Paper I). The full list of emission lines used, together with the corresponding ionization potentials are given in Table 2. Line identifications and typical spectra during the various outburst phases can be found in Cassatella et al. (2004).

The intensity of the emission lines has been measured in individual spectra through interactive procedures, taking great care to avoid saturated data or regions affected by particle events or camera artefacts. 
Table 1. Basic parameters of CO novae

\begin{tabular}{lrrrr}
\hline \hline Nova & $t_{\text {disc. }}$ & $t_{0}$ & $V_{\max }$ & \multicolumn{1}{c}{$t_{3}$} \\
\hline V1668 Cyg 1978 & 3762 & 3763 & 6.1 & $23 \pm 3^{1}$ \\
PW Vul 1984 & 5909 & 5917 & 6.4 & $100 \pm 20^{2}$ \\
OS And 1986 & 6770 & 6773 & 6.2 & $20 \pm 3^{3}$ \\
N LMC 1988\#1 & 7242 & 7244 & 10.7 & $40 \pm 2^{4}$ \\
N LMC 1991 & 8365 & 8372 & 8.8 & $8 \pm 3^{5}$ \\
N LMC 1992 & 8937 & 8938 & 10.2 & $18 \pm 3^{6}$ \\
V705 Cas 1993 & 9329 & 9333 & 6.6 & $90 \pm 15^{7}$ \\
\hline
\end{tabular}

Notes: $t_{\text {disc. }}$ and $t_{0}$ are, respectively, the dates of the discovery and of the visual maximum expressed as Julian Day -2440000.

References: (1) Duerbeck et al. (1980) (see also Cassatella et al. 1979); (2) Gehrz et al. (1988); (3) Schwarz et al. (1997b); (4) Schwarz et al. (1998); (5) from the $V$ light curve in Della Valle (1991); (6) estimated by us from IAU circulars and FES photometry; (7) Mason et al. (1998).

Table 2. Line identifications.

\begin{tabular}{lrrl}
\hline \hline Ion & $\lambda$ & $\chi_{\text {up }}(\mathrm{eV})$ & Comments \\
\hline Mg II & 2800 & 7.64 & UV 1 \\
C II & 1335 & 11.26 & UV 1 \\
O I & 1300 & 13.60 & UV 2; pumped by H $L_{\beta}$ \\
C III] & 1909 & 24.38 & \\
$\mathrm{~N} \mathrm{III}]$ & 1750 & 29.60 & \\
{$[\mathrm{O}$ III] } & 1660 & 35.18 & \\
$\left.\mathrm{~N} \mathrm{IV}_{\mathrm{IV}}\right]$ & 1487 & 47.43 & \\
$\mathrm{~N} \mathrm{~V}$ & 1240 & 77.47 & \\
\hline
\end{tabular}

\section{Individual objects}

The temporal evolution of the listed emission line fluxes for the novae in our sample is shown in Fig. 1 (V1668 Cyg), Fig. 2 (OS And, V705 Cas and PW Vul) and in Fig. 3 (N LMC 1991, N LMC 1992 and N LMC 1998 \#1).

\subsection{V1668 Cyg}

V1668 Cygni is the first nova intensively monitored with the IUE satellite, and the first for which coordinated observations were secured over a very wide wavelength range, from the ultraviolet to the infrared (see Stickland et al. 1981 and references therein). The IUE observations cover a period of 838 days counted from the day of visual maximum (1978 September 11 or JD 2443762). Because of its intensive monitoring, this nova will be used as a reference for the evolution of the emission lines in the other novae of the present sample.

Figure 1 shows the evolution of the following emission lines, in order of increasing ionization potential: Mg II $2800 \AA$, C II $1335 \AA$, O I $1300 \AA$, C III] $1909 \AA$, N III] $1750 \AA$, O III] $1663 \AA$, N IV] $1487 \AA$, and N v $1240 \AA$. Filled circles represent measurements that can be ascribed to the above transitions. Open circles indicate that the emission is not due to the transition labeled in the plot, but to other transitions appearing at about the same wavelength in low resolution spectra. This is the case, for example, of the feature around $1750 \AA$ which, during

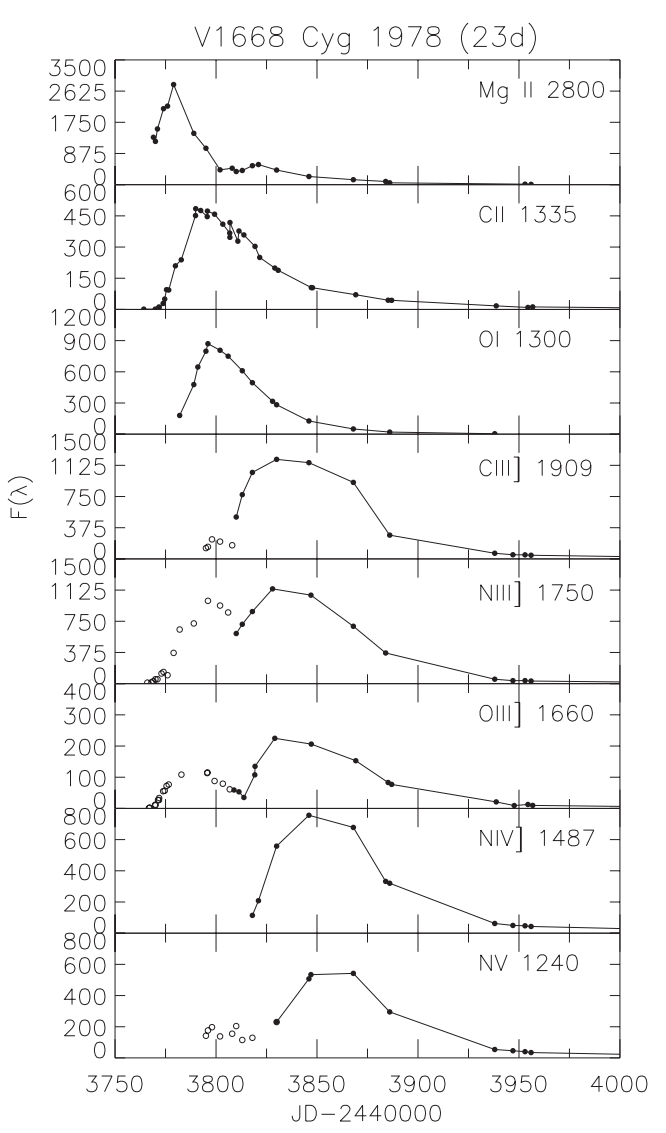

Fig. 1. Time evolution of the UV emission lines in V1668 Cyg. Open circles indicate that the emission is not due to the labeled ion (see text). Fluxes are expressed in units of $10^{-13} \mathrm{erg} \mathrm{cm}^{-2} \mathrm{~s}^{-1}$.

the earliest outburst phases, is not due to N III] $1750 \AA$ but to a complex pseudo-emission structure on top of which several narrow absorption lines from the N II multiplets UV 3, 4 and 5 are seen (Cassatella \& González-Riestra 1988).

\subsection{OS And, V705 Cas, PW Vul}

The IUE monitoring of these novae was not as detailed as that of V1668 Cyg; this explains why only a few emission lines could be followed in detail. Figure 2 indicates that the general spectral evolution of these objects follows the same trends as in V1668 Cyg.

OS And is a fast nova ( $t_{3}=20$ days) with a visual light curve similar to that of V1668 Cyg (see Kikuchi et al. 1988; Schwarz et al. 1997b). Kikuchi et al. (1988) reported that the degree of optical polarization increased from $0.78 \%$ to $1.73 \%$ from day 2 to day 22, and ascribed this to the formation of a dust shell. Although Gehrz et al. (1988) did not report any accompaniyng increase of the IR emission, the local minimum with an amplitude of $\approx 1.5$ mag in the visual light curve around day 35 seems to confirm that dust formation did actually take place. A detailed spectroscopic study of OS And based on IUE observations and a comparison with NLTE syntethic spectra is presented by Schwarz et al. (1997b). 

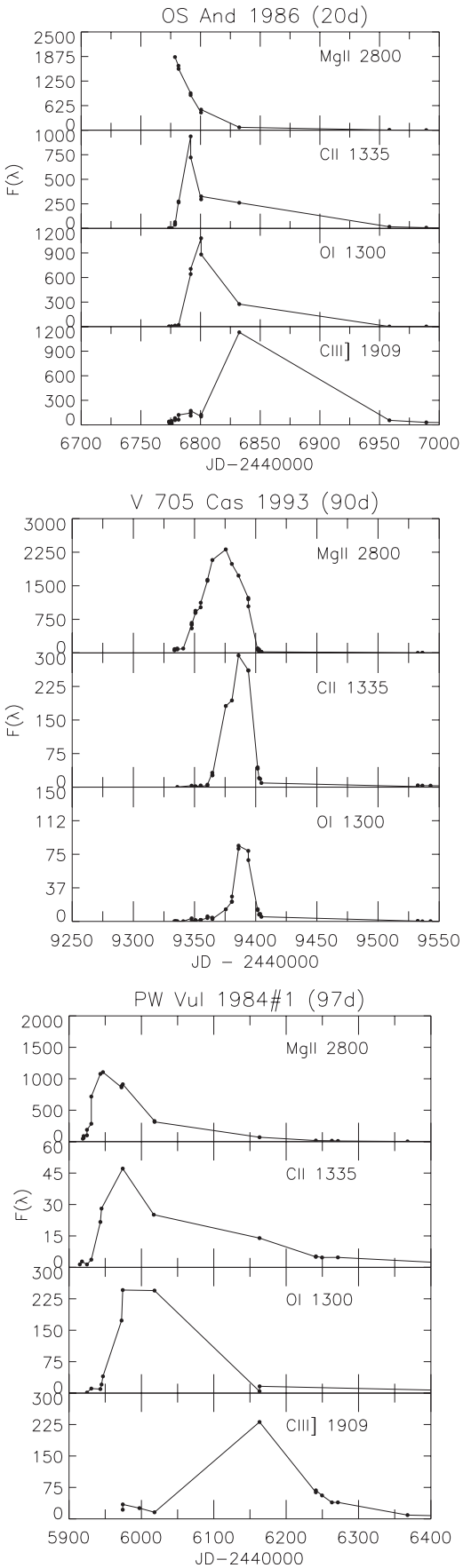

Fig. 2. Time evolution of the UV emission lines in OS And, V705 Cas and PW Vul. Open circles indicate that the emission is not due to the labeled ion (see text). Fluxes are expressed in units of $10^{-13} \mathrm{erg} \mathrm{cm}^{-2} \mathrm{~s}^{-1}$.

Nova V705 Cas $\left(t_{3}=90\right.$ days $)$ is well known for the spectacular dust formation episode observed after day 62 as a steep decline of the visual light curve (Evans et al. 1996). The formation of an optically thick carbon dust shell was confirmed by Mason et al. (1998), who reported an increase of the infrared flux coinciding in time with the deep (about 6 mag) minimum of the visual light curve observed around day 105. As pointed out by Mason et al. (1998), the early photometric behaviour of V705 Cas is similar to that of the intermediate polar old nova
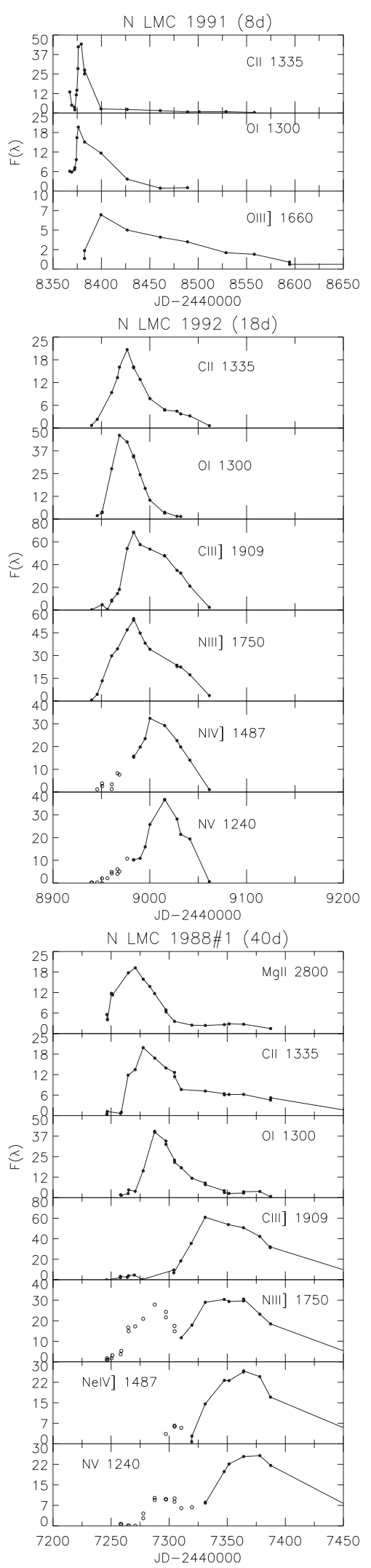

Fig. 3. Time evolution of the UV emission lines in the LMC Novae. Open circles indicate that the emission is not due to the labeled ion (see text). Fluxes are expressed in units of $10^{-13} \mathrm{erg} \mathrm{cm}^{-2} \mathrm{~s}^{-1}$.

DQ Her 1935. A description of the spectral evolution in the UV before dust formation is provided by Hauschildt et al. (1994). 
Table 3. Times of maximum line emission, $\Delta t=t_{\max }($ ion $)-t_{0}$, days.

\begin{tabular}{|c|c|c|c|c|c|c|c|c|c|}
\hline Nova & $t_{3}$ & Mg II & C II & O I & C III] & $\mathrm{N}$ III] & O III] & N IV] & $\mathrm{Nv}$ \\
\hline N LMC 91 & 8 & & $5 \pm 1$ & $4 \pm 2$ & & & $27 \pm 22$ & & $90 \pm 31$ \\
\hline N LMC 92 & 18 & & & $32 \pm 8$ & $45 \pm 7$ & & & $62 \pm 5$ & $77 \pm 14$ \\
\hline OS And & 20 & & $18 \pm 10$ & $27 \pm 20$ & $59 \pm 30$ & & & & \\
\hline V1668 Cyg & 23 & $16 \pm 6$ & $29 \pm 7$ & $36 \pm 5$ & $67 \pm 14$ & $74 \pm 9$ & $83 \pm 25$ & $83 \pm 25$ & $95 \pm 10$ \\
\hline N LMC 88\#1 & 40 & $26 \pm 6$ & $33 \pm 6$ & $42 \pm 10$ & $87 \pm 16$ & $103 \pm 10$ & & $120 \pm 12$ & $134 \pm 12$ \\
\hline V705 Cas & 90 & $43 \pm 8$ & $56 \pm 7$ & $\geq 61$ & & & & & \\
\hline PW Vul & 97 & $30 \pm 14$ & $56 \pm 20$ & $96 \pm 20$ & $246 \pm 120$ & & & & \\
\hline
\end{tabular}

The effects of the dust formation episode in V705 Cas were large enough to also affect the UV continuum flux at $1455 \AA$, which suffered from a drastic drop around day 60 (see Fig. 3 in Paper I). It appears from Fig. 2 that a similar drop is present in the flux in the O I $1300 \AA$ line. For this reason only a lower limit of about 60 days can be given for the date of the O I flux maximum.

$\mathrm{PW}$ Vul $\left(t_{3}=100\right.$ days $)$ is the slowest nova in our sample. Its light curve during early outburst is very similar to that of DQ Her (see Gehrz et al. 1988) and of V705 Cas, but it does not show any evidence of dust formation. According to Gehrz et al. (1988) the absence of dust could be due to the low metal abundance, to an early ionization of the ejecta, or to the density in the shell being low enough to prevent efficient grain formation. The ultraviolet spectrum of this nova during the nebular phase has been studied by Andreä et al. (1991). Recent determinations of chemical abundances in PW Vul can be found in Schwarz et al. (1997a).

\subsection{The three $L M C$ novae}

LMC 1991 is a fast nova with a decay time $t_{3}=8$ days, sensibly shorter than the other two LMC novae LMC 1992 $\left(t_{3}=18\right.$ days, close to that of V1668 Cyg) and LMC 1988\#1 $\left(t_{3}=40\right.$ days). It is the brightest nova so far observed in the Large Magellanic Cloud, and is peculiar for his low metallicity and enhanced CNO abundance, as reported by Schwarz et al. (2001). According to these authors, LMC 1991 could belong to a small sub class of novae termed super-bright novae studied by Della Valle (1991) in the M31 galaxy and in the Virgo cluster.

According to Schwarz et al. (1997b) the evoultion of the UV continuum and emission lines of Nova LMC 1992 are very similar to that of the galactic nova OS And. Despite this similarity, no dust formation has been reported for LMC 1992.

LMC 1988\#1 has been extensively observed in the UV, optical and infrared bands; its photometric behaviour is very similar to that of V1668. On day 55 after visual maximum it developed a thin dust shell which was detectable for about two months (Schwarz et al. 1998).

In summary, the evolution of the UV emission lines shown in Figs. 1-3 reveal the following common features:

- The maximum line emission gradually shifts from low to high ionization lines.

- The evolution to higher ionization conditions is faster for faster novae.
- High ionization emission lines tend to remain strong for a longer time than low ionization lines.

\section{Delay of the flux maximum in the emission lines}

We have shown in the previous section that there is a common pattern governing the rate of growth of the degree of ionization in novae. To proceed further in a more quantitative way we have measured, in each nova, the time $\Delta t=\left(t_{\max }(\right.$ ion $\left.)-t_{0}\right)$ needed for a given line to reach flux maximum, counted from the date of visual maximum $t_{0}$ given in Table 1 . For lines showing a well-defined flux maximum, the time of maximum was taken as the observed one and the error as the the half distance between the previous and the following observation. In some cases, however, due to the poor time coverage of the observations, a flux plateau was observed instead of a well defined line maximum (see e.g. the N V line in V1668 Cyg and the O I line in PW Vul). In these cases we have assumed that the maximum took place half-way between the beginning and the end of the plateau and, as associated error, we have taken the half length of the plateau.

The measuremts of $\Delta t$ for the different emission lines, in order of increasing ionization potential, are reported in Table 3 together with the corresponding errors and the number of measurements. Using these data we show in Fig. 4 the dependence of $\Delta t$ on the ionization potential $\chi_{\text {ion }}$ for six of the novae considered (V 705 Cas is not shown because of the small interval in ionization potential covered).

Figure 4 shows in a quantitative way what was already anticipated in the previous section, namely that $\Delta t$ is an increasing function of $\chi_{\text {ion }}$, i.e. the higher the degree of ionization of a line, the later the emission maximum occurrs. It also appears from Fig. 4 that, for a given line, the time $\Delta t$ needed for a nova to reach a maximum emission is longer for novae with larger $t_{3}$. In the $\mathrm{C} \mathrm{III]}$ line, for example, $\Delta t$ ranges from 45 days for $\mathrm{N} \mathrm{LMC} 1992\left(t_{3}=18\right.$ days) to 246 days for PW Vul $\left(t_{3}=97\right.$ days $)$.

Since the degree of ionization increases with time at a faster rate for faster novae, we have normalized the time delay of the emission line maximum to the $t_{3}$ time and computed, for each ion, the weighted mean of $\Delta t / t_{3}$ for the seven novae of the sample. These values are plotted in Fig. 5 and reported in Table 4 together with the corresponding rms errors. One can easily appreciate from Fig. 5 that the mean values of $\Delta t / t_{3}$ are quite close to each other despite the wide range of $t_{3}$ values. Given this 


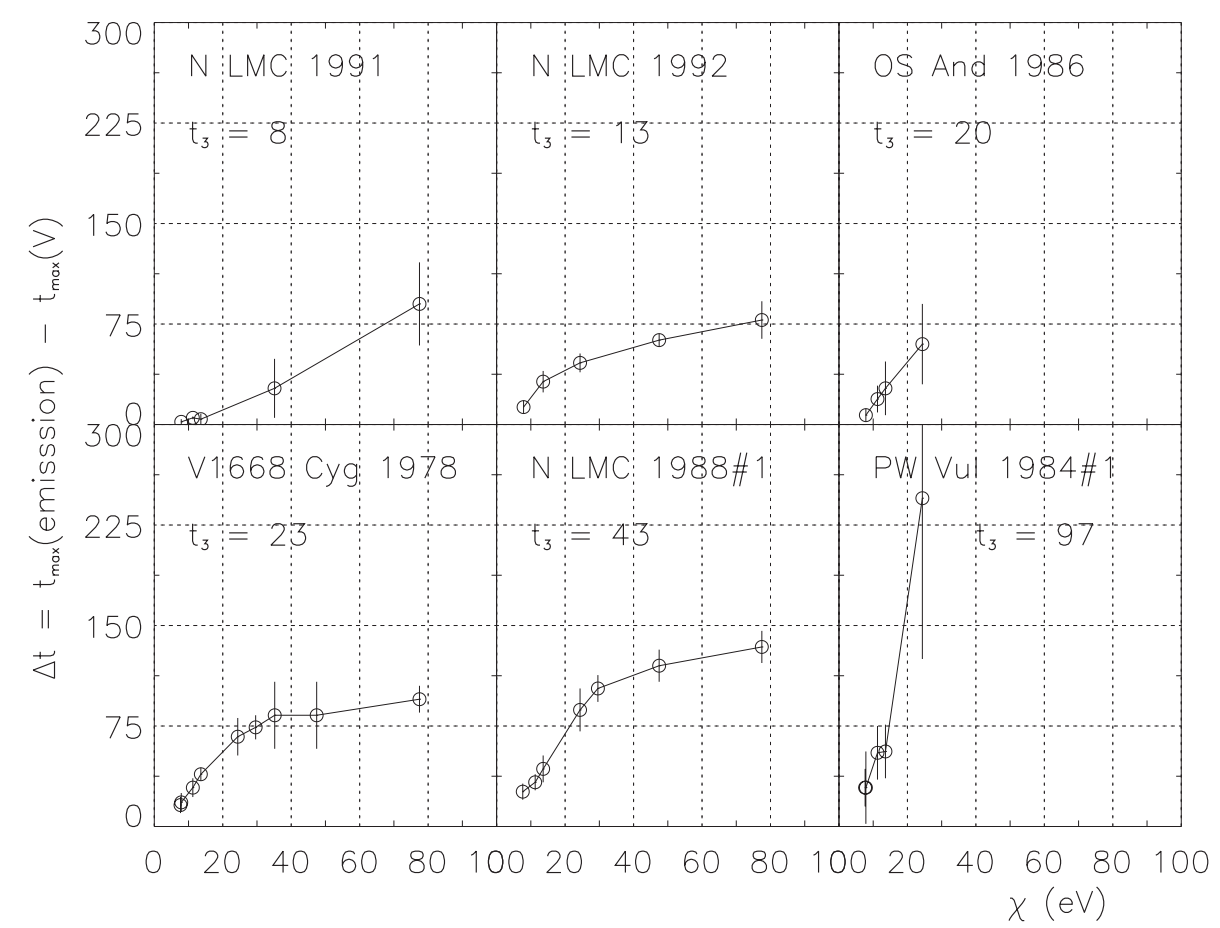

Fig. 4. Delay of the emission line flux maximum with respect to the time of visual maximum as a function of the ionzation potential for six novae in our sample.

Table 4. Normalized time of maximum flux.

\begin{tabular}{lrrrrr}
\hline \hline Ion & $\lambda$ & $\chi_{\text {up }}(\mathrm{eV})$ & $\Delta t / t_{3}$ & rms & $N$ \\
\hline Mg II & 2800 & 7.64 & 0.48 & 0.07 & 4 \\
C II & 1335 & 11.26 & 0.67 & 0.06 & 6 \\
O I & 1300 & 13.60 & 1.10 & 0.11 & 6 \\
C III] & 1909 & 24.38 & 2.45 & 0.25 & 5 \\
N III] & 1750 & 29.60 & 2.76 & 0.21 & 2 \\
O III] & 1660 & 35.18 & 2.89 & 0.35 & 2 \\
N IV] & 1487 & 47.43 & 3.25 & 0.20 & 3 \\
N V & 1240 & 77.47 & 3.70 & 0.23 & 4 \\
\hline
\end{tabular}

homogeneous pattern, one can empirically define the following three phases describing the evolution of the UV emission lines:

The initial phase. As a consequence of the expansion, the pseudophotosphere recedes to the inner and hotter regions, while the ejected envelope becomes more and more transparent to the ionizing photons. This process is observed as a progressive hardening of the UV continuum which endures for a time of the order of $t_{3}$. After this time, the UV continuum becomes temporarily cooler due to the increasing contribution from the hydrogen recombination continuum, which peaks around 2800-3000 $\AA$ in the IUE range (Paper I). This clear signature of the increased level of ionization goes in step with the strengthening of the O I $1300 \AA$ line (this line is pumped via fluorescence by hydrogen Ly $\beta$, and therefore reflects the strength of the Ly $\beta$ emission). We define as the initial phase the one of high to moderate opacity in the continuum which precedes the maximum

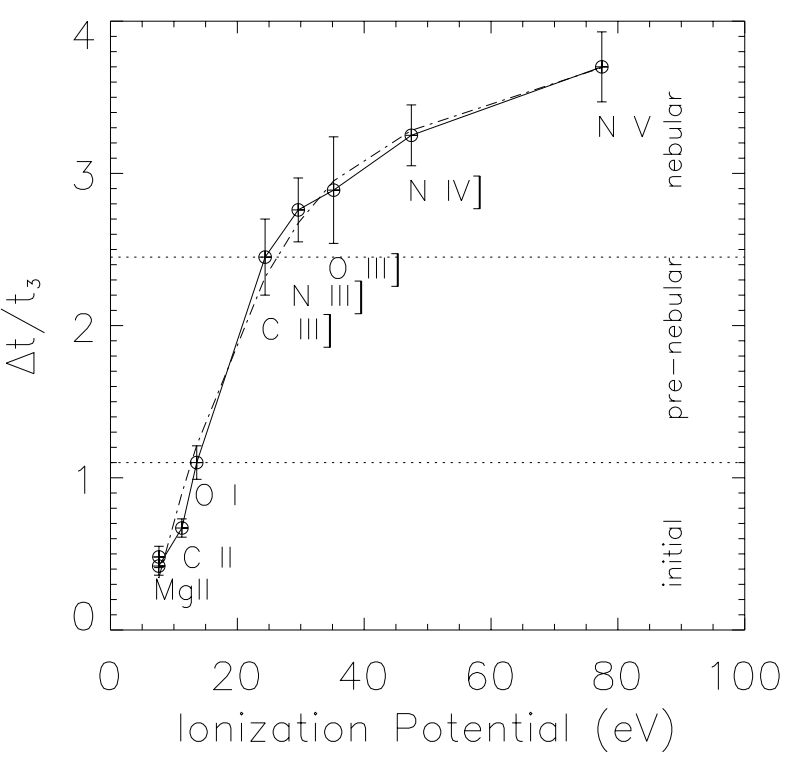

Fig. 5. Normalized delay time of the emission line maximum as a function of line ionization potential (see data in Table 4). The figure reports also the different phase of development. The dashed-dotted curve represents a $3 \mathrm{rd}$ order polynomial fit to the data.

emission in the O I $1300 \AA$ line. The O I maximum takes place at $\Delta t \approx 1.1 t_{3}$ (see Table 4$)$.

The pre-nebular phase. After the O I $1300 \AA$ emission maximum, the degree of ionization in the nebula increases at about the same rate as before. This, together with the decreased density in the emitting regions, favours the appearence and increase in strength of the C III] $1909 \AA$ A intercombination line. We define as the pre-nebular phase the one in between the flux maxima of the O I and the C III] 
lines. The C III] maximum takes place at $\Delta t \approx 2.5 t_{3}$ (see Table 4).

The nebular phase. During this phase, which starts after the $\mathrm{C}$ III] maximum, the degree of ionization increases at a faster rate than before. In addition to intercombination lines and resonance lines with higher levels of ionization, a number of forbidden lines (not seen before) are also observed.

\section{Discussion and conclusions}

In this paper we have studied how the degree of ionization of the UV emission lines increases with time after a nova event and have provided a quantitative definition of three different regimes of spectral development: the initial phase, the prenebular phase and the nebular phase, as depitcted in Fig. 5. The progressive increase of the ionization level shown in this figure was predicted by Bath (1978; see his Fig. 5) in the context of the optically thick model of classical novae.

The present study has interesting implications for the open problem of the nuclear turn-off time in novae. We have shown that the time $\Delta t$ needed for a given emission line to reach a flux maximum is proportional to the $t_{3}$ time, so that $\Delta t / t_{3} \approx$ constant. This means that faster novae reach the conditions of maximum ionization before slower novae. Since emission line fluxes are good tracers of the temperature and luminosity changes of the post-nova, one should expect that also the nuclear turnoff time is proportional to $t_{3}$ in the present sample of CO novae. A similar result has been obtained for the $\mathrm{ONeMg}$ novae studied by Vanlandingham et al. (2001).

The most remarkable feature of Fig. 5 is that the $\Delta t$ data, once scaled for $t_{3}$, all collapse onto a single curve. A way to understand such a homologous behaviour is to consider the equation of continuity of a stationary wind expanding at a velocity $v$ from a star. At a radial distance $r$ from the center of the star the mass loss rate $\dot{M}$ is:

$\dot{M}=4 \pi r^{2} \rho v$

where $\rho$ is the wind density. If the expansion velocity of the wind is constant with radius, a shell forms in which the density varies as $\rho=\rho_{0} r_{0}^{2} / r^{2}$, where $r_{0}$ and $\rho_{0}$ are the radius and density at the base of the wind, respectively. Such conditions are reached, for example, when the velocity in the wind has stabilized to a value close to that of the terminal velocity. In the case of nova V1974 Cyg, this happens after a time which is of the order of the $t_{3}$ time (Cassatella et al. 2004a).

Integration of Eq. (1) over a time interval $\Delta t$ provides the mass of the shell $M_{\mathrm{s}}$ :

$M_{\mathrm{s}}=4 \pi r_{0}^{2} \rho_{0} v \Delta t$.

We assume now that $t_{3} \propto v^{-2}$ (McLaughlin 1960). Dividing both members of Eq. (2) by $t_{3}$ and considering that $\Delta t / t_{3}$ is approximately constant for a given emission line (see Table 4) we have:

$\frac{\Delta t}{t_{3}} \propto \frac{M_{\mathrm{s}} v}{4 \pi r_{0}^{2} \rho_{0}} \approx$ const.

According to the white dwarf models by Hamada \& Salpeter (1961), the product $r_{0}^{2} \rho_{0}$ at the photosphere does not vary by
Table 5. Literature and estimated ejected masses.

\begin{tabular}{|c|c|c|c|}
\hline Nova & $v_{\exp }$ & $M_{\mathrm{s}}$ (lit.) & $M_{\mathrm{s}}$ (est.) \\
\hline N LMC 91 & $1670^{(1)}$ & $\begin{array}{rr}3 \times 10^{-4} & (7) \\
1.2 \times 10^{-6} & (8)\end{array}$ & $2.6 \times 10^{-5}$ \\
\hline N LMC 92 & $1500^{(2)}$ & & $2.9 \times 10^{-5}$ \\
\hline OS And & $1220^{(1)}$ & & $3.6 \times 10^{-5}$ \\
\hline V1668 Cyg & $1160^{(3)}$ & $3.8 \times 10^{-5}$ & \\
\hline N LMC 98\#1 & $1800^{(4)}$ & & $2.4 \times 10^{-5}$ \\
\hline V705 Cas & $840^{(5)}$ & $\approx 10^{-5} \quad$ & $5.2 \times 10^{-5}$ \\
\hline PW Vul & $750^{(6)}$ & $\begin{array}{r}1.6 \times 10^{-4} \\
3.2 \times 10^{-6} \\
3 \times 10^{-4} \text { to } 3 \times 10^{-2}\end{array}$ & $5.9 \times 10^{-5}$ \\
\hline
\end{tabular}

References: (1) Our estimate from the Mg II line in IUE spectra; (2) Our estimate from the FWZI of the N IV] emission line observed with HST-GHRS on Jan. 7, 1993, as reported by Shore (1998; Fig. 5), see also Shore (1992); (3) Cassatella et al. (1979); (4) Schwarz et al. (1998); (5) Wooward \& Greenhouse (1993), and our estimate from the Mg II line in IUE spectra; (6) Cassatella \& González-Riestra (1988); (7) Schwarz et al. (2001). (8) Williams (1994). (9) mean value from Stickland et al. (1981) and Gehrz et al. (1980); (10) Mason et al. (1998); (11) Schwarz et al. (1997a), see text; (12) Gehrz et al. (1988); (13) Saizar et al. (1991).

more than a factor of 3 in the mass range 0.8 to $1.2 M_{\odot}$. If the base of the wind is at the white dwarf photosphere, we can then consider $r_{0}^{2} \rho_{0} \approx$ constant as a reasonable approximation. Provided the filling factor of the shell is the same for all novae considered, it follows from Eq. (3) that $M_{\mathrm{s}} v \approx$ constant, i.e.:

$M_{\mathrm{s}} \propto\left(M_{\mathrm{s}} v\right)^{*} / v$

where the asterisk refers to a nova, to be used as the reference, for which reliable determinations are available for the ejected mass and the expansion velocity. A suitable reference object is the well-studied nova V1668 Cyg, for which two consistent determinations of the ejected mass are available from the literature: $M_{\mathrm{S}}=5.53 \times 10^{-5} M_{\odot}($ Stickland et al. 1981) and $M_{\mathrm{s}}=2 \times 10^{-5} M_{\odot}(\mathrm{Gehrz}$ et al. 1980). We will adopt the mean value: $M_{\mathrm{s}}^{*}=3.8 \times 10^{-5} M_{\odot}$. For the expansion velocity, we have adopted $v^{*}=1160 \mathrm{~km} \mathrm{~s}^{-1}$ corresponding to the principal system absorption lines on day 8 after discovery (Cassatella et al. 1979).

We can at this point test the validity of the assumptions made by comparing the shell mass determinations available from the literature with the values predicted from the expansion velocity through Eq. (4) and taking V1668 Cyg as a reference. The shell masses so obtained are reported in Table 5 together with the literature data $M_{\mathrm{s}}$ and $v$ for the novae in our sample.

From Table 5, the predicted shell mass is 3 times smaller than in the literature for PW Vul, and 5 times smaller for V705 Cas while, in the case of N LMC 1991, the shell mass is about a factor of 10 smaller than in Schwarz et al. (2001), and about 3 times larger than the value obtained from the O I shell mass (Williams 1994), supposing that this latter represents $15 \%$ of the total shell mass. Such discrepancies are not surprising because, from one side, "observed" shell masses are extremely uncertain (see the very wide spread of values found for PW Vul 
and N LMC 1991 in Table 5) and, from the other side, the present semiempirical model is heavily limited by the poor knowledge of the velocity and density distribution within the line emitting regions and by the physical conditions at the base of the nova winds.

Again taking V1668 Cyg as a reference, we can now use Eq. (3) to estimate the shell masses of OS And and N LMC 1988\#1 from the observed expansion velocities in Table 5. The values so obtained, reported in Table 5, are quite similar for the two objects.

Both Eq. (3) and observations (Table 5) indicate that slow novae possess smaller expansion velocities and larger shell masses than fast novae: the shell mass of the slowest novae of our sample (V705 Cas and PW Vul) appears to be about a factor of 2 larger than faster novae. Such a trend is consistent with the predictions of the multicycle evolution models of Prialnik and Kovetz (1995), with the relationship $M_{\mathrm{s}} \propto v^{2} t_{3}^{2}$ reported by Shore (2002), and is also implicit in the correlation between $M_{\mathrm{s}}$ and the decay time $t_{2}$ found by Della Valle et al. (2002). Further studies are needed to model in a proper way the dependence of $M_{\mathrm{s}}$ on the expansion velocity and on other fundamental parameters such as the white dwarf temperature, the accretion rate etc.

We believe that no substantial progress can be made in assessing the problem of the ejected mass and of the nuclear turnoff time in novae unless an adequate observational effort is made to determine accurately their expansion velocity law. In particular, it is very important to determine the asymptotic expansion velocity at least at a time $\Delta t \approx 2-3 t_{3}$. In addition, since most ejected mass determinations rely on the distance to the nova, extended investigations on novae in nearby galaxies should be highly encouraged.

Acknowledgements. We are grateful to the Referee of this paper, Dr. A. Evans, for his interesting suggestions and to Dr. M. Friedjung for comments.

\section{References}

Andreä, J., Drechsel, M. A. J., Snijders, M. A. J., \& Cassatella, A. 1991, A\&A, 244, 111

Bath, G. T. 1978, MNRAS, 182, 35

Cassatella, A., Benvenuti, P., Heck, A., et al. 1979, A\&A, 74, L18
Cassatella, A., \& González-Riestra, R. 1988, Physics of Formation of Fe II Lines Outside LTE, ed. R. Viotti, et al. (D. Reidel), 115

Cassatella, A., Altamore, A., \& González-Riestra, R. 2002, A\&A, 384, 1023 (Paper I)

Cassatella, A., Lamers, H. J. G. M. L, Rossi, C., Altamore, A., \& González-Riestra, R. 2004a, A\&A, 420, 571

Cassatella, A., González-Riestra, R., \& Selvelli, P. L. 2004b, Classical Novae, INES Access Guide No. 3, ESA Special Report 1283

Della Valle, M. 1991, A\&A, 252, L9

Della Valle, M., Pasquini, L., Daou, D., \& Williams, R. E. 2002, A\&A, 390,155

Duerbeck, W., Rindermann, K., \& Seitter, W. C. 1980, A\&AS, 81, 157

Evans, A., Geballe, T. R., Rawlings, J. M. C., \& Scott, A. D. 1996, MNRAS, 282, 1049

Gehrz, R. D., Hackwell, J. A., Grasdalen, G. L., et al. 1980, ApJ, 239, 570

Gehrz, R. D., Harrison, T. E., Ney, E. P., et al. 1988, ApJ, 329, 894

Hamada, T., \& Salpeter, E. E. 1961, ApJ, 264, 1041

Hauschildt, P. H., Starrfield, S., Shore, S. N., et al. 1994, AJ 108, 1008

Kikuchi, S., Kondo, M., \& Mikami, Y. 1988, PASP, 40, 491

Livio, M. 1992, ApJ, 393, 516

Mason, C. G., Gehrz, R. D., Woodward, C. E., et al. 1998, ApJ, 494, 783

McLaughlin, D. B. 1960, in Stars and Stellar Systems VI, ed. J. L. Greenstein, Stellar Atmospheres, (Chicago: Univ. Chicago Press), 585

Prialnik, D., \& Kovets, A. 1995, ApJ, 445, 789

Saizar, P., Starrfield, S., Ferland, G. J., et al. 1991, ApJ, 367, 310

Schwartz, G. J., Starrfield, S., Shore, S. N., \& Hauschildt, P. H. 1997a, MNRAS, 290, 75

Schwartz, G. J.,Hauschildt, P. H., Starrfield, S., et al. 1997b, MNRAS, 284, 669

Schwarz, G. J., Hauschildt, P. H., Starrfield, S., et al. 1998, MNRAS, 300,931

Schwarz, G. J., Shore, S. N., Starrfield, S., et al. 2001, MNRAS, 320, 103

Shore, S. N. 1992, IAU Circ. 5657

Shore, S. N. 1998, The scientific Impact of the Goddard High Resolution Spectrograph, ed. J. C. Brandt, T. B. Ake, C. C. Petersen, ASP Conf. Ser., 143, 113

Shore, S. N., 2002, in Classical Nova Explosions, ed. M. Hernanz, J. José, AIP, 175

Stickland, D. J., Penn, C. J., Seaton, M. J., Snijders, M. A. J., \& Storey, P. J. 1981, MNRAS, 197, 107

Vanlandingham, K. M., Schwarz, G. J., Shore, S. N., \& Starrfield, S. 2001, ApJ, 121, 1126

Williams, R. E. 1994, ApJ, 426, 279

Woodward, C. E., \& Greenhouse, M. A. 1993, IAU Circ., 5910 